\title{
Check-list dei mammiferi nella Riserva naturale di Monticchie (Lodi; Italia settentrionale) - 1985-2012
}

\section{Vincenzo Ferri ${ }^{1}$, Christiana Soccini ${ }^{1}$, Corrado Battisti ${ }^{2}$}

\author{
${ }^{1}$ Studio associato Natura Arcadia, Via Valverde 4, 01016 Tarquinia (Viterbo), Italia. \\ E-mail: progetto.chirotteri@gmail.com; studio.natura.arcadia@gmail.com \\ ${ }^{2}$ Corresponding author: Corrado Battisti, Via dei Carafa 274, 00148 Roma, Italia. E-mail: c.battisti@provincia.roma.it
}

\begin{abstract}
Riassunto - Relativamente ad una area protetta della bassa pianura lodigiana (Riserva naturale di Monticchie, Lodi), è stata ottenuta una check-list di mammiferi su un arco temporale relativamente ampio (1985-2012). La lista (39 specie) comprende alcuni taxa rari e localizzati nella pianura padana (es., alcuni chirotteri, Arvicola amphibius, Sorex minutus, Neomys anomalus, Micromys minutus, Muscardinus avellanarius), perché legate ad ambienti specifici (es., ambienti umidi) e/o sensibili alla frammentazione forestale. Vengono riportate alcune evidenze sulla possibile scomparsa locale di Arvicola amphibius e sulla recente espansione di specie sia alloctone (Myocastor coypus, Sylvilagus floridanus, Procyon lotor) sia autoctone (Sciurus vulgaris, Sus scrofa, Capreolus capreolus).
\end{abstract}

\begin{abstract}
A long-term local mammal check-list for Monticchie (Lodi, northern Italy) - 1985-2012.

Data obtained form a large time span allow to obtain a heterogeneous and rich check-list of mammals (39 species) with rare, localized and fragmentation-sensitive species (Chiroptera, Arvicola amphibius, Sorex minutus, Neomys anomalus, Micromys minutus, Muscardinus avellanarius) mixed to introduced game and/or allochtonous species. In the last decades, we obtained evidences for a possible local extinction of Arvicola amphibius, for introduction of some allochtonous species (Myocastor coypus, Sylvilagus floridanus, Procyon lotor), and for the range expansion of further autochtonous species (Sciurus vulgaris, Sus scrofa, Capreolus capreolus).
\end{abstract}

Key-words: check-list, extinction/colonization dynamics, alien species, protected area, northern Italy.

\section{INTRODUZIONE}

Le check-list relative a determinati gruppi tassonomici costituiscono il primo livello informativo utile a valutare il grado di naturalità e il livello ecologico e conservazionistico di un sito. Tale conoscenza può rivelarsi di particolare importanza nelle aree naturali protette: grazie a tali informazioni possono essere, infatti, avviate specifiche indagini a livello di specie (es., minacciate o, al contrario, problematiche) o definite appropriate misure di gestione e conservazione. In particolare, se i dati sono stati ottenuti su lunghe serie temporali può anche essere possibile individuare pattern specifici e dinamiche in atto (es., di estinzione-colonizzazione; Sutherland, 2000).
I mammiferi terrestri comprendono un gran numero di taxa di interesse sia conservazionistico sia gestionale (es., specie alloctone problematiche) che, negli ultimi decenni, sono state caratterizzate da marcate dinamiche demografiche e cambiamenti nei pattern di abbondanza e distribuzione a scala locale, regionale, globale (cfr. Boitani et al., 2003; Amori et al., 2008). A tal proposito, la redazione di check-list locali, ottenute per siti specifici e su lunghi periodi temporali possono evidenziare, relativamente a questo gruppo, processi e dinamiche in atto, sottolineando anche cambiamenti nello stato dell'ambiente (es., incremento di degrado o, al contrario, di naturalità di un sito).

Scopo di questo contributo è quello di fornire un inquadramento della teriofauna di un'area protetta della bassa pianura lodigiana (Italia settentrionale), ottenuto dall'analisi della letteratura e di dati originali raccolti localmente e in modo eterogeneo nell' arco di oltre 25 anni.

\section{MATERIALI E METODI}

La Riserva di Monticchie (Somaglia, Lodi), istituita dalla Regione Lombardia con D.G.R. n. 53446 del 18 giugno 1985 e ratificata con D.G.R. n. IV/1177 del 28 luglio 1988, dal 1995 compresa tra i Siti di Importanza Comunitaria e con D.G.R. 3624/2006 confermata tra le Zone di Protezione Speciale (SIC e ZPS IT2090001), si estende per 238 ha, di cui circa 24 godono di massima tutela (Canova, 2012; Ferri \& Canova, 2011; cfr. anche Agapito Ludovici \& Cecere, 1999). La Riserva è situata nella bassa pianura lodigiana, lungo il terrazzo geologico del Po e a circa 2,5 chilometri dall'attuale corso del fiume. I suoli originano da alluvioni formate da ghiaie, sabbie e limi. Ad eccezione delle scarpate del terrazzo fluviale, l'area è prevalentemente pianeggiante, con dislivelli minimi (altitudine tra 47 e $60 \mathrm{~m}$ s.l.m.) che consentono, grazie alla presenza di una falda freatica abbastanza superficiale, il differenziarsi della vegetazione secondo aspetti caratterizzati dal diverso grado di igrofilia: boschi igrofili a Salix alba e Alnus glutinosa; boschi meso-igrofili a dominanza di Quercus robur; arbusteti 
a Cornus sanguinea; vegetazione a idrofite dei corsi d'acqua e incolti igrofili a struttura erbacea (Zavagno, 2003).

Buona parte della superficie della Riserva è caratterizzata da seminativi coltivati prevalentemente a mais. Gli ontaneti della riserva sono fra i più estesi della pianura lombarda e le canalizzazioni interne derivano da fontanili di terrazzo (Pollini, 1980; Cotta Ramusino et al., 2000). La sovrastante pianura lodigiana è caratterizzata da campi variamente riquadrati delimitati da fossi, cavi e rogge irrigue accompagnati da filari di pioppi o salici, grandi cascine isolate, insediamenti organizzati intorno a sistemi di corte o a preesistenze castellane (Cavallini, 2007).

L'interesse ecologico dell'area deriva dalla presenza di biotopi forestali sia naturali (vegetazione arborea ripariale) sia di tipo artificiale (rimboschimenti a pioppo, Populus sp.) che hanno mantenuto caratteristiche di naturalità medio-alta.

Per quanto riguarda l'area vasta circostante spicca il corridoio fluviale del Po che in questo tratto lodigiano è caratterizzato dalla presenza di sistemi naturali di notevole valore ecologico comprendendo oltre a Monticchie, altre quattro Zone di Protezione Speciale (ZPS: IT2090702 Po di Corte S. Andrea, IT2090501 "Senna Lodigiana", IT2090701 "Po di San Rocco al Porto", IT2090503 “Castelnuovo Bocca d'Adda"). La golena del Fiume Po Lodigiano è stata anche inserita dalla Regione Lombardia tra le Aree Prioritarie per la Biodiversità (n. 25 'Fiume Po', segnalata come area di Primo Livello della Rete Ecologica Regionale, Bogliani et al., 2007).

Tra la fascia golenale e la Riserva di Monticchie si collocano infrastrutture viarie particolarmente trafficate e di difficile attraversamento per la piccola fauna terricola: l'Autostrada A1 Milano-Bologna e la nuova linea ferroviaria veloce TAV MI-BO (Canova, 2012).

L'area è stata fatta oggetto di indagini anche per altri gruppi di vertebrati e invertebrati, specialmente anfibi e uccelli (es., Ferri, 1988; Ferri et al., 1995; Ferri \& Agapito Ludovici, 2002; Ferri \& Soccini, 2005b; Ferri, 2006; Ferri \& Canova, 2011; Canova, 1989; Barbieri \& Bernini, 2004; Fasola et al., 2007).

Sono state raccolte tutte le informazioni bibliografiche e i dati originali (sia opportunistici, sia standardizzati) disponibili per l'area nel periodo 1985 - 2012. La prima campagna di ricerche sui mammiferi della Riserva naturale è stata svolta mediante diverse tecniche di rilevamento a metà degli anni '80 (campionamento sia con snap trap sia con trappole "a vivo"; Canova \& Saino 1986, Canova, 1992). Successivamente sono state svolte ricerche occasionali specificatamente rivolte a gruppi che hanno richiesto tecniche e metodi specifici riguardanti in particolare:

- i micro-mammiferi terricoli: maggio-settembre 1990, utilizzando pitfalls e live traps tipo Sherman prodotte dalla ditta Tavecchio di Milano; settembre 1985 - settembre 1986 e maggio - settembre 1988: utilizzando pitfalls (V. Ferri, dati non pubblicati);

- i micro-mammiferi terricoli attraverso l'analisi di borre di strigiformi (prevalentemente di Asio otus, ma anche di Strix aluco e Tyto alba, raccolte in diversi mesi del 2003 e del 2012, V. Ferri e C. Soccini, dati non pubblicati);
- i chirotteri: attraverso campionamenti con rilevatori elettronici di ultrasuoni e successive analisi bioacustiche per il riconoscimento delle specie; con bat-detector Pettersson D200x, D240x e D1000x e con bat-detector AnaBat II Titley nei seguenti periodi: marzo - settembre 2003; maggio - agosto 2010; maggio - settembre 2011 e 2012 (Soccini \& Ferri, 2012); ulteriori ricerche sono state effettuate con bat-detector da incaricati della Provincia di Lodi (AA.VV., 2004). Infine una serie di osservazioni occasionali non standardizzate hanno consentito di ottenere informazioni su altre specie di meso- e macromammiferi (AA.VV., 2004; V.Ferri e C.Soccini, dati non pubblicati).

Tutti i dati di presenza sono stati vagliati criticamente e associati all'anno di primo e ultimo rilevamento. Nella check-list delle specie presenti non è stata considerata l'eventuale attribuzione sottospecifica. Ad ogni specie è stata associata l'informazione relativa all'inserimento in determinate categorie di minaccia IUCN e negli Allegati della Direttiva 92/43/CEE "Habitat". La nomenclatura e l'ordine sistematico sono tratte da Boitani et al. (2003), Amori et al. (2008) e Lanza (2012).

\section{RISULTATI}

Nel periodo 1985-2012 nell'area di studio sono state ottenute evidenze di presenza per 39 specie di mammiferi appartenenti a 7 Ordini (Tab. 1). Per una specie segnalata in passato in modo aneddotico per l'area di studio, $M u$ stela putorius, non si sono avuti riscontri né diretti né indiretti e pertanto non è stata considerata in questo lavoro. Due specie sono risultate di interesse conservazionistico più elevato perché inserite nella nuova lista IUCN con le categorie di minaccia Near Threatened (Myotis oxygnathus e Oryctolagus cuniculus); 10 specie sono inserite in almeno uno degli Allegati della Direttiva 79/409/CEE "Habitat" (9 chirotteri e un roditore, Muscardinus avellanarius; Tab. 1).

\section{DISCUSSIONE}

L'elevata eterogeneità ambientale, con presenza di ambienti peculiari (es., aree umide, frammenti forestali residui), spiega la teriofauna relativamente numerosa e diversificata dell'area di studio, soprattutto se comparata con siti limitrofi (Prigioni et al., 2011; Ottolini \& Aceto, 1996). Sono state rilevate nel periodo di studio sia specie stenoecie e stenotope, indicative quindi di una buona qualità ambientale e in declino a scala regionale (es., Neomys anomalus, Micromys minutus, Sorex minutus, Arvicola amphibius, Myodes glareolus; per quest'ultimo cfr. Nieder et al. 1987), sia sensibili alla frammentazione (es., Muscardinus avellanarius; Spinozzi et al. 2011), sia, infine, legate a mosaici ambientali ancora relativamente intatti, che mostrano popolazioni fortemente discontinue e frammentate nel territorio padano (es., Meles meles, Martes foina; Canova \& Rosa, 1993, Vigorita \& Cucé, 2008). I chirotteri sono rappresentati da 8 specie (Ferri \& Soccini, 2005, AA.VV. 2004, Ferri \& Soccini, 2012). 
Tab. 1 - Check-list dei mammiferi della Riserva naturale di Monticchie (Lodi) (Ordine, Famiglia, Specie). È stato riportato il livello di interesse conservazionistico delle specie (IUCN: categorie di minaccia IUCN: LC = Least Concern, NT = Near Threatened; Habitat: specie inserita in determinati allegati della Direttiva 92/43CEE). Note: (1) Crani e resti scheletrici di entrambe le specie in borre di Asio otus (V. Ferri, dati originali 2003), in passato erano assegnati a Sorex araneus; (2) sulla Scheda Natura2000 è segnalato Neomys fodiens; la carcassa rinvenuta nel 1988, assegnata a N. anomalus, è conservata oggi presso il Centro Museale di Penna S. Andrea (TE); (3) segnalata unicamente nel 1995; (4) Introdotta a fini venatori nel 2006; (5) Prima segnalazione nel 2007; conferma fotografica nel 2008; (6) Non più segnalata dal 1994; (7) Prima segnalazione nel 1989; (8) prima segnalazione nel 2010; (9) Prima segnalazione ottobre 2010; (10) Prima segnalazione maggio 2011.

\begin{tabular}{|c|c|c|c|}
\hline & IUCN & Habitat & Note \\
\hline \multicolumn{4}{|l|}{ ORDINE ERINACEOMORPHA } \\
\hline \multicolumn{4}{|l|}{ Famiglia Erinaceidae } \\
\hline Riccio europeo Erinaceus europaeus Linnaeus, 1758 & $\mathrm{LC}$ & & \\
\hline \multicolumn{4}{|l|}{ ORDINE SORICOMORPHA } \\
\hline \multicolumn{4}{|l|}{ Famiglia Talpidae } \\
\hline Talpa comune Talpa europaea Linnaeus, 1758 & $\mathrm{LC}$ & & \\
\hline \multicolumn{4}{|l|}{ Famiglia Soricidae } \\
\hline Toporagno nano Sorex minutus Linnaeus, 1766 & $\mathrm{LC}$ & & \\
\hline Toporagno comune Sorex antinorii Bonaparte, 1840 & $\mathrm{LC}$ & & (1) \\
\hline Toporagno di Arvonchi Sorex arunchi Lapini \& Testone, 1998 & LC & & (1) \\
\hline Toporagno acquatico di Miller Neomys anomalus Cabrera, 1907 & $\mathrm{LC}$ & & $(2)$ \\
\hline Crocidura ventre bianco Crocidura leucodon (Hermann, 1780) & $\mathrm{LC}$ & & \\
\hline Crocidura minore Crocidura suaveolens (Pallas, 1811) & $\mathrm{LC}$ & & \\
\hline \multicolumn{4}{|l|}{ ORDINE CHIROPTERA } \\
\hline \multicolumn{4}{|l|}{ Famiglia Vespertilionidae } \\
\hline Vespertilio di Daubenton Myotis daubentonii (Kuhl, 1817) & $\mathrm{LC}$ & IV & \\
\hline Vespertilio maggiore Myotis myotis (Borkhausen, 1979) & $\mathrm{LC}$ & II, IV & \\
\hline Vespertilio di Monticelli Myotis oxygnathus (Monticelli, 1885) & NT & II, IV & \\
\hline Pipistrello nano Pipistrellus pipistrellus (Schreber, 1774) & $\mathrm{LC}$ & IV & \\
\hline Pipistrello albolimbato Pipistrellus kuhlii (Kuhl, 1817) & $\mathrm{LC}$ & IV & \\
\hline Pipistrello di Savi Hypsugo savii (Bonaparte, 1817) & $\mathrm{LC}$ & IV & \\
\hline Nottola comune Nyctalus noctula (Schreber, 1774) & $\mathrm{LC}$ & IV & (3) \\
\hline Serotino comune Eptesicus serotinus (Schreber, 1774) & $\mathrm{LC}$ & IV & \\
\hline Orecchione bruno Plecotus auritus (Linnaeus, 1758) & $\mathrm{LC}$ & IV & \\
\hline \multicolumn{4}{|l|}{ ORDINE LAGOMORPHA } \\
\hline \multicolumn{4}{|l|}{ Famiglia Leporidae } \\
\hline Coniglio selvatico Oryctolagus cuniculus (Linnaeus, 1758) & NT & & \\
\hline Lepre europea Lepus europaeus Pallas, 1778 & $\mathrm{LC}$ & & \\
\hline Minilepre Sylvilagus floridanus (J.J. Allen, 1890) & - & & (4) \\
\hline
\end{tabular}




\begin{tabular}{|c|c|c|c|}
\hline & IUCN & Habitat & Note \\
\hline \multicolumn{4}{|l|}{ ORDINE RODENTIA } \\
\hline \multicolumn{4}{|l|}{ Famiglia Sciuridae } \\
\hline Scoiattolo comune Sciurus vulgaris Linnaeus, 1758 & $\mathrm{LC}$ & & (5) \\
\hline \multicolumn{4}{|l|}{ Famiglia Gliridae } \\
\hline Moscardino Muscardinus avellanarius (Linnaeus, 1758) & $\mathrm{LC}$ & IV & \\
\hline \multicolumn{4}{|l|}{ Famiglia Cricetidae } \\
\hline Arvicola acquatica Arvicola amphibius (Linnaeus, 1758) & $\mathrm{LC}$ & & (6) \\
\hline Arvicola campestre Microtus arvalis (Pallas, 1778) & $\mathrm{LC}$ & & \\
\hline Arvicola del Savi Microtus savii (De Sélys Longchamps, 1838) & $\mathrm{LC}$ & & \\
\hline Arvicola rossastra Myodes glareolus (Schreber, 1780) & LC & & \\
\hline \multicolumn{4}{|l|}{ Famiglia Muridae } \\
\hline Topo selvatico Apodemus sylvaticus (Linnaeus, 1758) & $\mathrm{LC}$ & & \\
\hline Topolino delle risaie Micromys minutus (Pallas, 1771) & $\mathrm{LC}$ & & \\
\hline Topo domestico Mus musculus Linnaeus, 1758 & $\mathrm{LC}$ & & \\
\hline Surmolotto Rattus norvegicus (Berkenhout, 1769) & - & & \\
\hline Ratto nero Rattus rattus (Linnaues, 1758) & $\mathrm{LC}$ & & \\
\hline \multicolumn{4}{|l|}{ Famiglia Myocastoridae } \\
\hline Nutria Myocastor coypus (Molina, 1782) & - & & (7) \\
\hline \multicolumn{4}{|l|}{ ORDINE CARNIVORA } \\
\hline \multicolumn{4}{|l|}{ Famiglia Canidae } \\
\hline Volpe comune Vulpes vulpes (Linnaeus, 1758) & $\mathrm{LC}$ & & \\
\hline \multicolumn{4}{|l|}{ Famiglia Mustelidae } \\
\hline Faina Martes foina (Erxleben, 1777) & $\mathrm{LC}$ & & \\
\hline Donnola Mustela nivalis Linnaeus, 1766 & $\mathrm{LC}$ & & \\
\hline Tasso Meles meles (Linnaeus, 1758) & $\mathrm{LC}$ & & \\
\hline Procione Procion lotor (Linnaeus, 1758) & - & & (8) \\
\hline \multicolumn{4}{|l|}{ ORDINE ARTIODACTYLA } \\
\hline \multicolumn{4}{|l|}{ Famiglia Suidae } \\
\hline Cinghiale Sus scrofa Linnaeus, 1758 & $\mathrm{LC}$ & & (9) \\
\hline \multicolumn{4}{|l|}{ Famiglia Cervidae } \\
\hline Capriolo Capreolus capreolus (Linnaeus, 1758) & $\mathrm{LC}$ & & $(10)$ \\
\hline
\end{tabular}


Oryctolagus cuniculus è risultato in contrazione numerica nel corso dell'indagine: le popolazioni di questa specie sono di autoctonia dubbia analogamente a quelle di Lepus europaeus, le cui introduzioni a scopo venatorio in gran parte dell'Italia settentrionale hanno pesantemente alterato la originaria struttura genetica (cfr. Angelici \& Spagnesi in Amori et al. 2008; Vigorita \& Cucé 2008).

Il lungo arco temporale nel quale si è svolta questa indagine ha consentito di ottenere evidenze per alcune probabili estinzioni locali e nuove colonizzazioni. In particolare, Sorex minutus e Arvicola amphibius non sono stati più rilevati, rispettivamente, dal 1990 e dal 1994: le due specie possono ritenersi localmente scomparse, pur se sarebbero necessarie indagini specifiche. Di Arvicola amphibius si conserva un individuo rinvenuto morto nella Riserva nel 1985 e tassidermizzato, nella collezione teriologica di V. Ferri. Tra i chirotteri, Nyctalus noctula, riportato nella scheda del S.I.C/Z.P.S., non è più stato segnalato dal 1995.

Al contrario alcune specie hanno colonizzato recentemente l'area: tra queste le alloctone Myocastor coypus (dal 1989), Procyon lotor (dal 2004) e Sylvilagus floridanus (dal 2006) e le autoctone Sciurus vulgaris (dal 2007), Sus scrofa (dal 2010), in fase espansiva dopo l'introduzione a fini venatori, e Capreolus capreolus (dal 2011), quest'ultima in colonizzazione spontanea lungo il fiume Po, presumibilmente dall'Appennino Piacentino.

In particolare Myocastor coypus, presente in Lombardia dal 1976 (oss. pers.: cfr. anche Gariboldi, 1993), è specie sottoposta nella provincia a campagne di controllo tramite trappolamenti ed i suoi danni alla vegetazione delle sponde e all'assetto idraulico della canalizzazione interna della Riserva sono per ora limitati (pur se esistono evidenze generali di un suo possibile impatto sulle popolazioni di uccelli acquatici: Angelici et al., 2012). Procyon lotor è stata recentemente segnalata (2004-2010) lungo il fiume Adda ( 1 ind. investito a ca. un $\mathrm{km}$ dal confine est della Riserva, nel 2010) (Canova \& Rossi, 2008).

Questa prima analisi sottolinea la necessità di avviare ulteriori indagini allo scopo di confermare, anche a livello quantitativo e di popolazione, le presenze e le assenze delle specie più sensibili $\mathrm{o}$, al contrario, problematiche (es., specie alloctone) in questo sito di elevato interesse conservazionistico.

\section{Ringraziamenti}

Gli Autori ringraziano per la collaborazione fornita tutta l'Amministrazione Comunale di Somaglia. Un particolare ringraziamento per l'aiuto logistico e nei rilevamenti in questi anni alla Dott.ssa Mariangela Quartieri. Molte segnalazioni derivano dalle preziose osservazioni e dalle intense ricerche svolte negli anni considerati nella Riserva di Monticchie da: Adriano Mascherpa, Alfredo Lucchini, Alex Coppi, Andrea Agapito Ludovici, Antonio Cremonesi, Bassano Riboni, Franco Zavagno, Francesco Ciossani, Giampiero Fumi, Giancarlo Quadrelli (alla memoria), Giovanni Barbieri, Luca Canova, Marina Mansi, Maurizio Sesenna, Oreste Maraboli, Paola Contardi, Paolo Lucchini, Rita Mabel Schiavo, Sara Filippini, Silvio Pirovano. Un ringraziamento alle famiglie Fioretti, Maz- zoleni, Ciserani, Toninelli, Rossi, Olivari che hanno permesso le ricerche dei roosts di chirotteri e di rapaci notturni presso gli annessi rurali nelle loro proprietà.

\section{BIBLIOGRAFIA}

AA.VV., 2004 - Monitoraggio degli habitat e della fauna nei pSIC della Provincia di Lodi. Servizio Fauna, Ambiente Naturale e Vigilanza Volontaria. Relazione interna. Provincia di Lodi, Lodi.

Agapito Ludovici A. \& Cecere F., 1999 - La Conservazione della natura nelle Oasi del Wwf Italia. Manuale di gestione. WWF Italia, Roma.

Amori G., Contoli L. \& Nappi A., 2008 - Mammalia II. Erinaceomorpha, Soricomorpha, Lagomorpha, Rodentia. Fauna d'Italia. Vol. 44. Calderini, Bologna.

Angelici C., Marini F., Battisti C., Bertolino S., Capizzi D. \& Monaco A., 2012 - Cumulative impact of rats and coypu on nesting waterbirds: first evidences from a small Mediterranean wetland (central Italy). Vie et Milieu - Life and Environment, 62: 137-141.

Barbieri F. \& Bernini F., 2004 - Distribution and status of Rana latastei in Italy (Amphibia, Ranidae). Italian Journal of Zoology, 71: 91-94.

Bogliani G., Agapito Ludovici A., Arduino S., Brambilla M., Casale F., Crovetto M.G., Falco R., Siccardi P. \& Trivellini G., 2007 - Aree prioritarie per la biodiversità nella pianura padana lombarda. Regione Lombardia e Fondazione Lombardia per l'Ambiente, Milano.

Boitani L., Lovari S. \& Vigna Taglianti A., 2003 - Mammalia III. Carnivora, Artiodactyla. Fauna d'Italia. Vol. 38. Calderini, Bologna.

Bontardelli L., Giordano V., Lazzarini M., Riservato E. \& Villa M., 2004 - Chirotteri, Lepidotteri e Odonati. In: AA.VV. 2004. Monitoraggio degli habitat e della fauna nei pSIC della Provincia di Lodi. Relazione interna. Servizio Fauna, Ambiente Naturale e Vigilanza Volontaria della Provincia di Lodi, Lodi.

Canova L., 1989 - Influence of snow cover on prey selection by Long-eared Owls Asio otus. Ethology Ecology \& Evolution, 1: 367-372.

Canova L., 1992 - Distribution and habitat preference of small mammals in a biotope of the north Italian plain. Bollettino di Zoologia, 59: 417-421.

Canova L., 1993 - Food habits and trophic relationships of small mammals in six habitats of the northern Po plain (Italy). Mammalia, 57: 189-199.

Canova L., 2012 - Monticchie compie 30 anni. La storia della riserva Naturale e i progetti ambientali. Amministrazione Comunale di Somaglia. Arti Graf. Solicitudo, Lodi.

Canova L. \& Rosa P., 1993 - Badger Meles meles and fox Vulpes vulpes food in agricultural land in the western Po Plain (Italy). Hystrix, Italian Journal of Mammalogy, 5: 73-78.

Canova L. \& Saino N., 1984 - I Mammiferi della Riserva "Monticchie". Relazione inedita per il WWF Sezione "Basso Lodigiano" e il Comune di Somaglia.

Canova L. \& Fasola M., 1991 - Communities of small mammals in six habitats of Northern Italy. Acta Theriologica, 36: 73-86. 
Canova L. \& Rossi S., 2008 - First records of the Northern Raccoon Procyon lotor in Italy. Hystrix, Italian Journal of Mammalogy, 19: 179-182.

Cavallini G., 2007 - Le case padronali del Lodigiano: esempi colti e umili. In: Cascine del lodigiano. Storia, vita e architettura di un paesaggio. AA.VV. Bolis Editore, Azzano San Paolo, 29-35.

Cotta Ramusino M., Longoni G. \& Seghetti C., 2000 - Le risorgive della riserva naturale di Monticchie (Somaglia, Lodi). Pianura, 12: 25-56.

Fasola M., Albanese G., Asoer, Boano G., Boncompagni E., Bressan U., Brunelli M., Ciaccio A., Floris G., Grussu M., Guglielmi R., Guzzon C., Mezzavilla F., Paesani G., Sacchetti A., Sanna M., Scarton F., Scoccianti C., Utmar P., Vaschetti G. \& Velatta F., 2007 Le garzaie in Italia, 2002. Avocetta, 31: 5-46.

Ferri V., 1988 - Anfibi e Rettili della Riserva Naturale "Monticchie": problematiche gestionali. Relazione inedita per il WWF Sezione "Basso Lodigiano".

Ferri V., 2006 - Aspetti di biologia riproduttiva di Rana latastei nel Sito di Importanza Comunitaria IT2090001 e Riserva naturale di Monticchie (Somaglia, Lodi). In: Atti del V Congresso Nazionale S.H.I., Calci (PI). Zuffi M.A.L. (ed.). Università degli Studi di Firenze, 69-73.

Ferri V. \& Agapito Ludovici A., 2002 - Monitoraggio delle popolazioni di Rana latastei di due riserve lombarde. Atti del $3^{\circ}$ Convegno "Salvaguardia Anfibi", Lugano. Cogecstre Edizioni, Penne, 63-70.

Ferri V., Agapito Ludovici A. \& Schiavo R. M., 1995 Problematiche di gestione delle popolazioni di Rana latastei delle Riserve Naturali Lombarde di "Monticchie" e "Le Bine". Quaderni della Civica Stazione di Idrobiologia, 19: 131-139.

Ferri V. \& Canova L., 2011 - Riproduzione della Rana di Lataste, Rana latastei, nella Riserva naturale orientata di Monticchie di Somaglia (Lombardia, Lodi). Pianura, 27: 174-177.

Ferri V. \& Soccini C., 2005a - Agricoltura e biodiversità. La tutela del Sito di Importanza Comunitaria "Monticchie" di Somaglia IT2090001. Comune di Somaglia, Lodi.
Ferri V. \& Soccini C., 2005b - Nuovi habitat per Rana latastei nel S.I.C. di Monticchie (IT2090001, Somaglia, Lodi). Comunicazione al PPP05 - Pond, Puddles and Pools, Trieste, 20-21 giugno 2005.

Gariboldi A., 1993 - La nutria (Myocastor coypus) in Lombardia. Supplementi di Ricerche di Biologia della Selvaggina, 21: 259-262.

Lanza B., 2012 - Fauna d'Italia. Mammalia V. Chiroptera. Calderini.

Nieder L., Varani C. \& Ambrogio A., 1987 - Prima segnalazione in Pianura padana di Chletrionomys glareolus. Hystrix, Italian Journal of Mammalogy, 2: 63-68.

Ottolini E. \& Aceto F., 1996 - La microteriofauna nelle riserve naturali della provincia di Cremona. Pianura, 8: 45-68.

Prigioni C., Balestrieri A. \& Remonti L., 2011 - I Mammiferi del Parco Adda Sud. I Libri del Parco Adda Sud, 5.

Soccini C. \& Ferri V., 2012 - Primi dati sulla chirotterofauna della golena del Po Lodigiano. In: VIII Congresso Italiano di Teriologia. Piacenza 9-11 Maggio 2012. Riassunti: Comunicazioni e Poster. Prigioni C., Balestrieri A., Preatoni D. G. \& Masseroni E. (eds.). Hystrix, Italian Journal of Mammalogy (N.S.) Suppl. 2012.

Spinozzi F., Battisti C. \& Bologna M. A., 2012 - Habitat fragmentation sensitivity in mammals: a target selection for landscape planning comparing two different approaches (bibliographic review and expert based). Atti della Accademia Nazionale dei Lincei. Rendiconti Lincei. Scienze Fisiche e Naturali, 23 (4): 365-373.

Sutherland W.J., 2000 - The Conservation Handbook. Blackwell Science, Massachussets.

Vigorita V. \& Cucé L., 2008 - La fauna selvatica in Lombardia. Rapporto 2008 su distribuzione, abbondanza e stato di conservazione di uccelli e mammiferi. Regione Lombardia, Università degli Studi di Milano Bicocca, Milano.

Zavagno F., 2003 - Osservazioni fenologiche sulla vegetazione di due riserve naturali della bassa pianura lombarda: Le Bine (CR-MN) e Monticchie (LO). Pianura, 17: 15-39. 